Original Article

\title{
Post Transplant Diabetes Mellitus in Ahmed Gasim Kidney Transplant Center, Sudan
}

\author{
Abdul-Rahman A El-Magzoub ${ }^{a^{*}}$, Sarra Elamin ${ }^{\mathrm{b}}$ \\ a. Consultant nephrologist, Ahmed Gasim Kidney Transplant Center, Khartoum, Sudan \\ b. Physician, Ahmed Gasim Kidney Transplant Center, Khartoum, Sudan
}

\begin{abstract}
Introduction: New onset post transplant diabetes mellitus (PTDM) is a common complication of kidney transplantation with a negative impact on patient and graft survival. Non-white ethnicity is a well known risk factor for PTDM.
\end{abstract}

Methods: This is a retrospective study of 221 Sudanese patients who received live donor kidney transplantation in Ahmed Gasim kidney transplant center between December 2001 and December 2007, focusing on the incidence, clinical course and prognosis of PTDM during the first post transplant year, and relating this to calcineurin inhibitor use.

Results: The 12 months cumulative incidence of PTDM in our transplant population was $17.6 \%$ and all cases required treatment with insulin. 53.8\% of PTDM cases were transient with a median duration of 76 days; by 12 months post transplant only $6.2 \%$ of patients were receiving insulin. The 12 months cumulative incidence of PTDM was higher in the tacrolimus compared to cyclosporine groups $(25.4 \%$ versus $6.6 \%$; OR $4.8,95 \%$ CI: $1.9-12.1 ; \mathrm{P}=0.00)$. During the follow up period $57.6 \%$ of PTDM patients in the tacrolimus group were able to discontinue insulin compared to $33.3 \%$ of PTDM patients in the cyclosporine groups (OR 2.7, 95\% CI: 0.4-17; $\mathrm{P}=0.3$ ). By 12 months post transplant, the proportion of patients receiving insulin was not significantly different between the two groups ( $8.0 \%$ versus $4.0 \%$; OR $2.1,95 \%$ CI $0.5-8.4 ; \mathrm{P}=0.2$ ).

Conclusion: The incidence of PTDM in our transplant population is not different from that reported in published randomized controlled trials and similarly, in the majority of cases PTDM was transient.

\footnotetext{
* Corresponding Author; Cardiac Surgery and Renal Transplant Center, Ahmed Gasim Hospital, PO Box 992, Khartoum North, Sudan. E.mail: aelmagzoub@yahoo.com
}

Key words: Post transplant diabetes mellitus, kidney transplantation, tacrolimus, cyclosporine, calcineurin inhibitor

\section{Introduction}

The development of diabetes mellitus after kidney transplantation was first described in 1964 by Starzl, who called it 'steroid diabetes'; Now, post transplant diabetes mellitus (PTDM) is recognized as being a common complication of kidney transplantation. $[1,2]$ The best method for determining the cumulative incidence of PTDM associated with the use of different immunosuppressive agents is from randomized controlled trials, but the results from these trials have been quite variable; $74 \%$ of this variability is explained by the type of immunosuppressive regimen. [1] The reported 12 months cumulative incidence of PTDM is $2-13.3 \%$ in patients receiving cyclosporine, azathioprine and prednisolone; $11.6-25.4 \%$ in patients receiving tacrolimus, azathioprine and prednisolone; and 4.7-12.2\% in patients receiving tacrolimus, mycophenolate mofetil and prednisolone. [1]

A number of large studies compared the cumulative incidence of PTDM in tacrolimus and cyclosporine treated patients, and showed that tacrolimus was associated with a higher relative risk of PTDM when compared to cyclosporine. [3,4] Large studies have also shown that PTDM is associated with a higher risk of patient mortality and graft loss, even when graft loss was censored for patient death. [1,3] Nevertheless, tacrolimus is associated with improved graft and patient survival, and with a significantly reduced risk of acute rejection and steroid resistant acute rejection. [3-5]

Besides calcineurin inhibitors, a number of risk factors for PTDM have been identified, including: non-white ethnicity, [1,3,6-8] pulse steroid treatment for acute rejection, [1] chronic hepatitis $\mathrm{C}$ virus infection (HCV) [3], and female gender [1,3]. 
Table 1: Characteristics of patients in the tacrolimus and cyclosporine groups

\begin{tabular}{llll}
\hline Characteristic & tacrolimus $(\mathrm{N}=130)$ & cyclosporine $(\mathrm{N}=91)$ & P value \\
\hline Age at transplant $($ mean $\pm \mathrm{SD})$ & $34 \pm 13$ years & $36 \pm 11$ years & 0.2 \\
Pediatric patients & $14.7 \%$ & $4.6 \%$ & 0.01 \\
Males & $73.8 \%$ & $80.2 \%$ & 0.2 \\
Chronic HCV infection & $4.2 \%$ & $10.4 \%$ & 0.08 \\
\hline
\end{tabular}

Some argue that it would be advisable to avoid the use of tacrolimus in patients who are already at high risk of PTDM because of non-modifiable risk factors, such as non-white ethnicity. However, those are the patients who are most likely to benefit from tacrolimus, because this is one of the factors known to impose a higher risk of graft loss.

Here we present a study of the incidence, clinical correlates and outcome of PTDM in a group of Sudanese patients, relating this to calcineurin inhibitor use. We hope to provide an insight into the best management of this common complication.

\section{Patients and Methods}

This is a retrospective study of a group of Sudanese patients who received living donor kidney transplantation at Ahmed Gasim kidney transplant center, Khartoum, Sudan, in the period from December 2000 to December 2007. Only patients who were diabetic before the transplant and patients who had primary graft non function were excluded from the analysis.

All patients received intravenous methyl prednisolone $500 \mathrm{mg}$ during the transplant surgery before removal of the vascular clamp, followed by a three drug regimen composed of cyclosporine (initial dose: $6-8 \mathrm{mg} / \mathrm{kg} / \mathrm{day}$, target trough level: $200-300 \mathrm{ng} / \mathrm{ml}$ reduced gradually to $100-150 \mathrm{ng} / \mathrm{ml}$ after 6 months) or tacrolimus (initial dose: $0.2-0.3 \mathrm{mg} / \mathrm{kg} / \mathrm{d}$, target trough level: $15-20 \mathrm{ng} / \mathrm{ml}$ reduced gradually to $5-7 \mathrm{ng} / \mathrm{ml}$ after 6 months) in addition to azathioprine $(1.5-2 \mathrm{mg} / \mathrm{kg} / \mathrm{d})$ and prednisolone (20-30 $\mathrm{mg} / \mathrm{d}$, reduced gradually to $5-7.5 \mathrm{mg} / \mathrm{d}$ after 3 months). Prior to February 2004, all patients were maintained on cyclosporine; as from February 2004, tacrolimus became the calcineurin inhibitor of choice. All patients received oral trimethoprim-sulphamethoxazole for the first 6 months after transplantation. Clinically diagnosed and/or biopsy proven acute rejection was treated with methylprednisolone $500 \mathrm{mg}$ daily for 3 days.

Data were collected by medical records review from the time of transplant until the end of the first post transplant year, the end of December 2007, death, or graft failure. Patients were divided into two groups according to the calcineurin inhibitor they used and examined for the presence or absence of PTDM and its clinical correlates.

PTDM was defined as two random blood glucose values $\geq 200 \mathrm{mg} / \mathrm{dl}$ and/or fasting blood glucose values $\geq 126$ $\mathrm{mg} / \mathrm{dl}$ taken on two separate occasions, in accordance with the definition of diabetes mellitus described by the WHO [9], and the international consensus guidelines on new-onset diabetes after transplantation [10]. Levels were confirmed over two weeks period. All such patients were treated with insulin. Resolution of PTDM was defined by fasting blood glucose values $\leq 115 \mathrm{mg} / \mathrm{dl}$ taken on two separate occasions without insulin or oral hypoglycaemic agents.

The 12 months cumulative incidence and clinical correlates of PTDM were studied and compared for the tacrolimus versus cyclosporine groups. PTDM was studied for possible association with patients age, gender, $\mathrm{HCV}$ infection, and the use of pulse steroids therapy for clinically diagnosed and/or biopsy proven acute rejection.

SPSS 15 for Windows was used to perform statistical analysis. Cross tabulation and the Chi square analysis was used to study possible risk factors and to test for their statistical significance. Results were considered statistically significant when $\mathrm{P}<0.05$.

\section{Results}

The study included 221 kidney transplant recipients, the great majority $(95.5 \%)$ received the kidneys from live related donors, and 10 patients $(4.5 \%)$ received the kidneys from live un-related donors. The mean age of patients was $35 \pm 12$ years (range: $8-65$ years), including 23 children $(10.4 \%)$ aged 18 years or less and the majority were males $(76.5 \%)$, Thirteen patients $(5.9 \%)$ had chronic HCV infection. The median duration of follow up was 29 months and $73.3 \%$ of patients had a minimum follow up of 12 months.

Ninety-one patients were maintained on cyclosporine while 130 patients were maintained on tacrolimus, three of whom were subsequently switched to cyclosporine during the first post transplant year because of suspected nephrotoxicity, gastrointestinal intolerance or bone 
Figure 1: Cumulative incidence of PTDM in the tacrolimus and cyclosporine groups

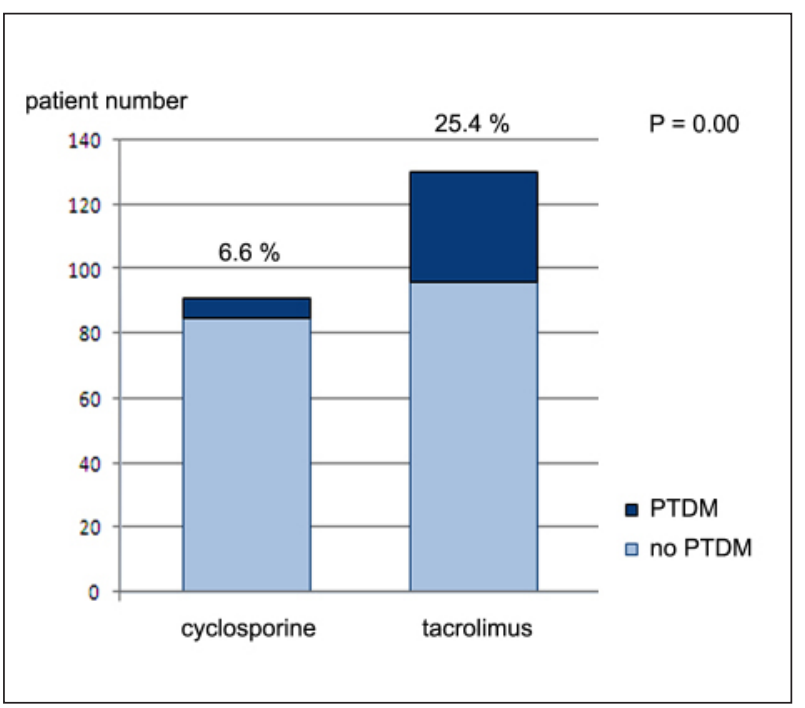

marrow suppression. Also, five patients from the cyclosporine group were switched from azathioprine to mycophenolate mofetil. The tacrolimus and cyclosporine groups were largely similar, but the tacrolimus group contained more paediatric patients $(14.7 \%$ versus $4.6 \%$, $\mathrm{P}=0.01)($ table 1).

PTDM was diagnosed in 39 patients, with an overall 12 month cumulative incidence of $17.6 \%$. The median time for diagnosis was 45 days post transplant, with $67.6 \%$ of cases being diagnosed within the first three months, and $94.6 \%$ of cases being diagnosed within the first six months. No cases of PTDM were diagnosed more than 7 months post transplant.

The overall prevalence of PTDM was 5.5\% (12/219) at one month post transplant, $11.7 \%(24 / 206)$ at three months post transplant, $11.6 \%(22 / 190)$ at six months post transplant, and $6.2 \%(10 / 162)$ at 12 months post transplant.

Overall, PTDM resolved in 21 of 39 patients during the follow up period $(53.8 \%)$. However, in 27 patients with PTDM who were followed up for 12 months or more, the resolution rate was $66.7 \%$. Transient PTDM had a median duration of 76 days (range: 16-270 days), it persisted for less than 3 months in $66.7 \%$ of cases and for less than 6 months in $90.5 \%$ of cases. Transient PTDM tended to occur later than persistent PTDM, with a median time of onset of 90 days versus 33 days post transplant.

All PTDM patients were treated by regular insulin, and $16 \%$ had at least one episode of diabetic ketoacidosis. PTDM patients needed re-hospitalization during the first post transplant year more often than did patients
Figure 2: Resolution rate of PTDM in the tacrolimus and cyclosporine groups

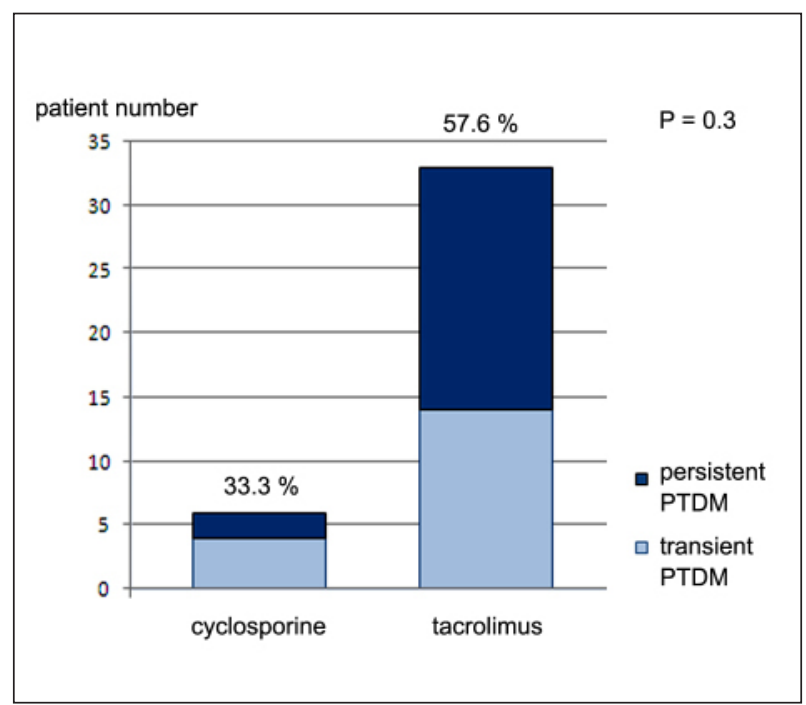

without PTDM (96.4\% versus $43.8 \%, \mathrm{P}=0.00)$, mainly for diabetes control or the treatment of infections.

The 12 months cumulative incidence of PTDM was higher for the tacrolimus compared to the cyclosporine groups (25.4\% versus 6.6\%; OR 4.8, 95\% CI: 1.9-12.1; $\mathrm{P}=0.00)$. PTDM tended to occur earlier in the tacrolimus group, with a median time of onset of 39 days post transplant compared to 75 days in the cyclosporine group (figure 1).

During the follow up period, the diabetic status was reversed in $57.6 \%$ of PTDM patients in the tacrolimus group compared to $33.3 \%$ of PTDM patients in the cyclosporine group (OR 2.7, 95\% CI: 0.4-17; $\mathrm{P}=0.3$ ) (figure 2). Among patients who had been follow up for 12 months or more, the diabetic status was reversed in $72.7(16 / 22)$ of PTDM patients in the tacrolimus group compared to $40 \%(2 / 5)$ of PTDM patients in the cyclosporine group (OR 4, 95\% CI: 0.5-30.2; $\mathrm{P}=0.2$ ).

The prevalence of PTDM was significantly higher in the tacrolimus compared to the cyclosporine group at one month post transplant $(8.5 \%$ versus $1.1 \%$; OR 8.1 , 95\% CI: $1-64.2 ; \mathrm{P}=0.02)$, at three months post transplant (16.3\% versus $4.8 \%$; OR 3.8, 95\% CI: $1.3-11.7$; $\mathrm{P}=0.01$ ), and at six months post transplant $(15.5 \%$ versus $6.3 \%$; OR 2.7, 95\% CI: 1-7.8; $\mathrm{P}=0.04)$. At 12 months post transplant, the difference in the prevalence of PTDM between the two groups was less marked and did not reach statistical significance $(8.0 \%$ versus $4.0 \%$; OR 2.1, 95\% CI 0.5-8.4; $\mathrm{P}=0.2$ ) (figure 3 ).

The PTDM and non-PTDM groups were compared for a number of possible risk factors. The only identified 
Figure 3: prevalence of PTDM at different time points post transplant

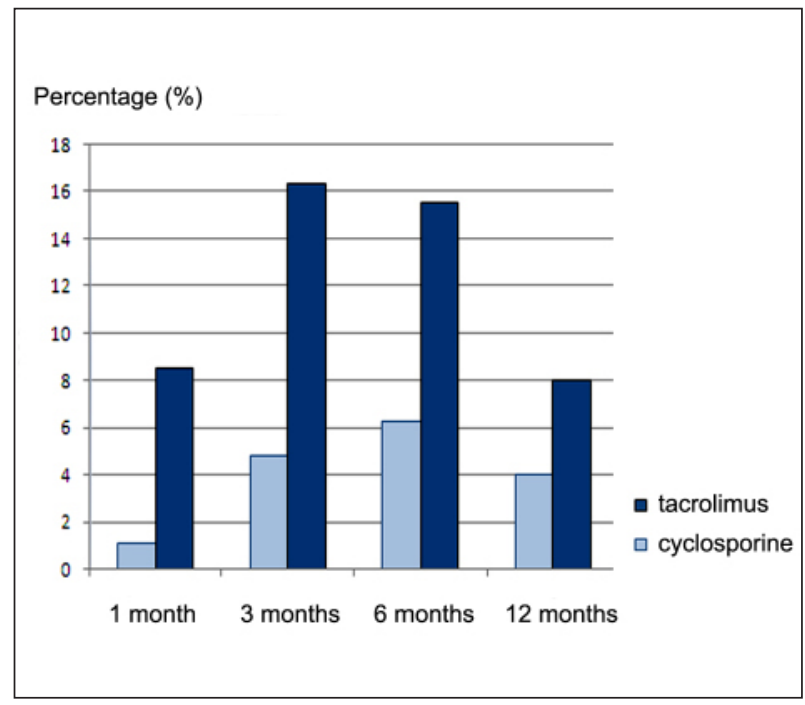

risk factors for PTDM were the use of tacrolimus versus cyclosporine $(25.4 \%$ versus $6.6 \%$; OR $4.8,95 \% \mathrm{CI}$ : $1.9-12.1 ; \mathrm{P}=0.00)$ and female gender $(26.9 \%$ versus 14.8\%; OR 2.1, 95\% CI: 1-4.5; $\mathrm{P}=0.04)$. Patients with chronic $\mathrm{HCV}$ infection tended to have a higher incidence of PTDM (38.5\% versus $16.8 \%$; OR 3.1, 95\% CI: $0.9-10.1 ; \mathrm{P}=0.07)$ as did patients who received at least one course of pulse steroid treatment for acute rejection during the first post transplant year $(25.9 \%$ versus 15.2\%; OR 2, 95\% CI: 0.9-4; $\mathrm{P}=0.06$ ); however, this association did not reach statistical significant. Pediatric age group was not associated with a higher incidence of PTDM (17.4\% versus 18.1\%; OR 1, 95\% CI: 0.3-3; $\mathrm{P}=0.6$ ). The incidence of PTDM among patients who had no episodes of acute rejection was $15.2 \%(25 / 164)$, the incidence of PTDM among patients who had one, two or three episodes of acute rejection within the first year was $21.3 \%(10 / 47), 42.9 \%(3 / 7)$ and $100 \%(1 / 1)$ respectively $(\mathrm{P}=0.03)$.

\section{Discussion}

The cumulative incidence of PTDM at 12 months posttransplant in our transplant population was $17.6 \%$; this is comparable to the incidence reported in the literature. A considerable proportion of PTDM cases resolved within the study period $(53.8 \%)$, and among patients who had been followed up for at least 12 months the resolution rate was $66.7 \%$; consequently, the prevalence of PTDM at 12 months post transplant was only 6.2\%. PTDM was associated with significant morbidity, the incidence of DKA was rather high (16\%), and all patients were treated with regular insulin. PTDM patients were much more likely to need hospital admission than patients who did not develop PTDM, mainly for diabetes control and the treatment of infections.

The cumulative incidence of PTDM in the cyclosporine group $(6.6 \%)$ is similar to the estimates reported from randomized controlled trials in patients receiving cyclosporine, azathioprine and prednisolone (2-13.3\%). [1] Similarly, the cumulative incidence of PTDM in the tacrolimus group (25.4\%) was within the reported range in randomized controlled trials in patients receiving tacrolimus, azathioprine and prednisolone (11.6-25.4\%). [1]

The cumulative incidence of PTDM in the cyclosporine group $(6.6 \%)$ is much lower than the incidence reported in a group of cyclosporine treated patients of Arab origin in a Kuwaiti center (29.6\%), which was partly attributed to the fact that Kuwait has a high background incidence of obesity and Type II diabetes mellitus [11]; the incidence was also lower than the incidence reported from a group of cyclosporine treated Saudi patients (41.4\%). [12]

The cumulative incidence of PTDM in the tacrolimus group $(25.4 \%)$ is also lower than the incidence reported by Hricik et al in a group of tacrolimus treated African American kidney transplant recipients (36\%). [13]

In this study, the use of tacrolimus compared with cyclosporine was associated with a significantly higher risk of PTDM (25.4\% versus $6.6 \%$; OR 4.8 , 95\% CI: $1.9-12.1 ; \mathrm{P}=0.000)$. The observed difference is consistent with the previous observation that despite the higher risk of PTDM attributed to non-white ethnicity, tacrolimus was associated with a lower relative risk of PTDM in non-white patients (29\% versus $7.8 \%$; RR 3.7) than white patients (12\% versus $1.2 \%$; RR 10.6$)$ when compared with cyclosporine. [14]

In this study, $57.6 \%$ of tacrolimus treated PTDM patients were able to discontinue insulin therapy during the study period, and among those who had been followed up for 12 months $72.7 \%$ were able to discontinue insulin therapy within the first post transplant year. In the US multicentre study, only $23.3 \%$ of tacrolimus treated PTDM patients discontinued insulin within the first year, and the rate of reversal of insulin dependence was only $41.2 \%$ by 5 years. [15] In the European multicentre study, $8.3 \%$ of tacrolimus treated patients developed insulin dependent PTDM, and by 12 months, the proportion of tacrolimus treated patients receiving insulin reduced to $5.5 \%$. [16]

In this study, $33.3 \%$ of cyclosporine treated PTDM patients were able to discontinue insulin therapy during the study period, and among those who had been followed up for 12 months $40 \%$ were able to discontinue insulin therapy within the first post transplant year. In the US multicentre study, only $16.7 \%$ of cyclosporine treated PTDM patients 
discontinued insulin within the first year. [15] Also, in the European multicentre study, 2.2\% of cyclosporine treated patients developed insulin dependent PTDM, and the same proportion $(2.2 \%)$ continued to receive insulin at 12 months. [16]

The use of tacrolimus and female gender were the only statistically significant risk factor for PTDM in our patient group, although chronic $\mathrm{HCV}$ infection and the need for at least one course of pulse steroid treatment for acute rejection during the first post transplant year had a clear tendency to be associated with PTDM. The incidence of PTDM increased significantly with the number of pulse steroid courses used during the first post transplant year.

All our cases of transient PTDM experienced resolution of their diabetes within 12 months of transplant, this concurs with Kiberd et al, who found that resolution occurred within six months for $94 \%$ of his patients. [17] If PTDM persists up to 12 months post transplant, it is unlikely to resolve; and switching to cyclosporine may be a valid therapeutic option; the reported success rate of this approach is (44-65\%). [18-20]

The choice of calcineurin inhibitor on the bases of diabetogenic risk should not be made at the expense of efficacy; measures such as steroid minimization or withdrawal and lower tacrolimus exposure may help maintain better long term survival.

\section{Conclusion}

The incidence of PTDM in our transplant population is no different from that reported in published randomized controlled trials, and a considerable proportion of cases are transient. The resolution rate among our patients was remarkable. The transient nature of most PTDM cases in our population mandates an individualized approach for management, so as not to deprive our patients from the well known benefits of tacrolimus on graft and patient survival.

\section{References}

1. Montori VM, Basu A, Erwin PJ, Velosa JA, Gabriel SE, Kudva YC. Post transplantation Diabetes: A systematic review of the literature. Diabetes Care. 2002;25:583-92.

2. Heisel O, Heisel R, Balshaw R, Keown P. New onset diabetes mellitus in patients receiving calcineurin inhibitors: a systematic review and meta-analysis. Am J Transplant. 2004 Apr;4(4):583-95.

3. Kasiske BL, Snyder JJ, Gilbertson D, Matas AJ. Diabetes mellitus after kidney transplantation in the United States. Am J Transplant. 2003 Feb;3(2):178-85.
4. Webster A, Woodroffe RC, Taylor RS, Chapman JR, Craig JC. Tacrolimus versus cyclosporine as primary immunosuppression for kidney transplant recipients. Cochrane Database Syst Rev. 2005 Oct 19;(4):CD003961.

5. Knoll GA, Bell RC. Tacrolimus versus cyclosporin for immunosuppression in renal transplantation: meta-analysis of randomised trials. BMJ. 1999 Apr 24;318(7191):1104-7.

6. Rodrigo E, de Cos MA, Fernández-Fresnedo G, Sánchez B, Ruiz JC, Piñera C, Palomar R, Cotorruelo JG, Gómez-Alamillo C, de Castro SS, de Francisco AL, Arias M. Higher initial tacrolimus blood levels and concentration-dose ratios in kidney transplant recipients who develop diabetes mellitus. Transplant Proc. 2005 Nov;37(9):3819-20.

7. Dooldeniya MD, Dupont PJ, He X, Johnson RJ, Joshi T, Basra R, Johnston A, Warrens AN. Renal transplantation in Indo-Asian patients in the UK. Am J Transplant. 2006 Apr;6(4):761-9.

8. Greenspan LC, Gitelman SE, Leung MA, Glidden DV, Mathias RS. Increased incidence in post-transplant diabetes mellitus in children: a case-control analysis. Pediatr Nephrol. 2002 Jan;17(1):1-5.

9. Alberti KG, Zimmet PZ. Definition, diagnosis and classification of diabetes mellitus and its complications. Part 1: diagnosis and classification of diabetes mellitus provisional report of a WHO consultation. Diabet Med. 1998 Jul;15(7):539-53.

10. Wilkinson A, Davidson J, Dotta F, Home PD, Keown P, Kiberd B, Jardine A, Levitt N, Marchetti P, Markell M, Naicker S, OConnell P, Schnitzler M, Standl E, Torregosa JV, Uchida K, Valantine H, Villamil F, Vincenti F, Wissing $\mathrm{M}$. Guidelines for the treatment and management of new-onset diabetes after transplantation. Clin Transplant. 2005 Jun;19(3):291-8.

11. Johny KV, Nampoory MR, Costandi JN, Gupta RK, Ninan VT, Samhan M, Muzairai I, Al-Mousawi M. High incidence of post-transplant diabetes mellitus in Kuwait. Diabetes Res Clin Pract. 2002 Feb;55(2):123-30.

12. Onwubalili JK, Obineche EN. High incidence of post-transplant diabetes mellitus in a single-centre study. Nephrol Dial Transplant. 1992;7(4):346-9.

13. Hricik DE, Anton HA, Knauss TC, Rodriguez V, Seaman D, Siegel C, Valente J, Schulak JA. Outcomes of African American kidney transplant recipients treated with sirolimus, tacrolimus, and corticosteroids. Transplantation. 2002 Jul 27;74(2):189-93. 
14. Cavaille-Coll MW, Elashoff MR. Commentary on a comparison of tacrolimus and cyclosporine for immunosuppression after cadaveric renal transplantation. Transplantation. 1998;65:142-5.

15. Vincenti F, Jensik SC, Filo RS, Miller J, Pirsch J. A long-term comparison of tacrolimus (FK506) and cyclosporine in kidney transplantation: evidence for improved allograft survival at five years. Transplantation. 2002 Mar 15;73(5):775-82.

16. Mayer AD. Chronic rejection and graft half-life: five-year follow-up of the European Tacrolimus Multicenter Renal Study. Transplant Proc. 2002 Aug;34(5):1491-2.

17. Kiberd M, Panek R, Kiberd BA. New onset diabetes mellitus post-kidney transplantation. Clin Transplant. 2006 Sep-Oct;20(5):634-9.
18. Bouchta NB, Ghisdal L, Abramowicz D, Broeders N, Surquin M, Hoang AD, Wissing KM. Conversion from tacrolimus to cyclosporin is associated with a significant improvement of glucose metabolism in patients with new-onset diabetes mellitus after renal transplantation. Transplant Proc. 2005 May;37(4):1857-60.

19. Oberholzer J, Thielke J, Hatipoglu B, Testa G, Sankary $\mathrm{HN}$, Benedetti E. Immediate conversion from tacrolimus to cyclosporine in the treatment of posttransplantation diabetes mellitus. Transplant Proc. 2005 Mar;37(2):9991000 .

20. Wyzgal J, Oldakowska-Jedynak U, Paczek L. Post transplantation diabetes mellitus under calcineurin inhibitor. Transplant Proc. 2003;35(6):2216-18. 